\title{
Effects of the Use of Non-Calcium Phosphate Binders in the Control and Outcome of Vascular Calcifications: A Review of Clinical Trials on CKD Patients
}

\author{
Piergiorgio Bolasco \\ Territorial Department of Nephrology and Dialysis, ASL, Cagliari, Via Turati 4/C1, 09045 Quartu Sant'Elena, Italy \\ Correspondence should be addressed to Piergiorgio Bolasco, pg.bolasco@tin.it
}

Received 12 April 2011; Accepted 13 April 2011

Academic Editor: Biagio Raffaele Di Iorio

Copyright ( 2011 Piergiorgio Bolasco. This is an open access article distributed under the Creative Commons Attribution License, which permits unrestricted use, distribution, and reproduction in any medium, provided the original work is properly cited.

\begin{abstract}
Vascular calcifications produce a high impact on morbidity and mortality rates in patients affected by chronic kidney disease and mineral bone disorder (CKD-MBD). Effects are manifested from the more advanced stages of CKD (stages 3-4), particularly in patients undergoing dialysis (CKD5D). In recent years, a large number of therapeutic options have been successfully used in the treatment of secondary hyperparathyroidism (SHPT), despite eliciting less marked effects on nonbone calcifications associated with CKD-MBD. In addition to the use of Vitamin D and analogues, more recently treatment with calcimimetic drugs has also been undertaken. The present paper aims to analyze comparative and efficacy studies undertaken to assess particularly the impact on morbidity and mortality rates of non-calcium phosphate binders. Moreover, the mechanism of action underlying the depositing of calcium and phosphate along blood vessel walls, irrespective of the specific contribution provided in reducing the typical phosphate levels observed in CKD largely at more advanced stages of the disease, will be investigated. The aim of this paper therefore is to evaluate which phosphate binders are characterised by the above action and the mechanisms through which these are manifested.
\end{abstract}

\section{Introduction}

Over the last 10 years, it has become increasingly apparent that chronic kidney disease-mineral and bone disorder (CKD-MBD) is associated to extra-skeletal manifestations, particularly cardiovascular calcifications in patients at the more advanced stages of CKD. The following manifestations have been observed: calcifications of the aorta, the carotid and the coronary arteries resulting in a reduced elasticity, diameter, compliance, and distensibility. The disease is not confined to central blood vessels but also involves the iliac-femoral axis and all arteries in the limbs, thus producing a high impact on the quality of life, morbidity and mortality of these patients [1-3]. An additional element implicated in the morbidity and mortality of patients is represented by the manifestation of calcifications on the endothelium of heart valves, in particular the mitral and aortic valves [4]. A fundamental impetus in the study of compression exerted by calcium deposits in the vessels was provided by Raggi using highly advanced procedures such as electron-beam computed tomography (EBCT), particularly to assess the involvement of coronary arteries [5]. Hyperphosphatemia is one of the main features of chronic kidney disease-mineral and bone disorder (CKD-MBD). An excess of phosphorus represents an independent risk factor for cardiovascular morbidity and mortality in patients with advanced CKD. Options available for use in the treatment of hyperphosphatemia are severe control of dietary phosphorus intake [6], use of phosphate binders [7], and optimized phosphorus output choosing high-efficiency dialysis as high flux-methodology [8-10]. Phosphate binders are used for their binding actions to reduce the absorption of phosphorus by the gastrointestinal lumen.

Calcium phosphate binders such as calcium carbonate or acetate are still widely used today. Evidence of an increased risk of onset and progression of nonbone, particularly vascular, calcifications has led to the use of caution by nephrologists, in spite of the markedly lower costs compared to the price of the new non-calcium. The concept whereby the at times excessive use of calcium-containing phosphate 
binders would appear to be widely accepted, together with the notion that in the future patients at the CKD5D stage should be treated to return dialysate calcium concentration to values below $1.5 \mathrm{mmol} / \mathrm{L}$ [11]. The latter is particularly important in patients undergoing peritoneal dialysis who are exposed at length to transfers of mass and therefore a longlasting, harmful positive calcium balance.

To briefly mention a few literature references cited in a recent review by Mason and Shepler [12], the authors underlined how seven of the nine studies reported a statistically significant increase in vascular calcification in patients taking calcium-containing phosphate binders as measured by coronary artery calcification scores and aortic calcification scores. Over the last year, the therapeutic target has changed; indeed, CKD-MBD may be characterized by SHPT, thus resulting in an increased risk when administering calciumbased phosphate binders in the presence of hypo-adinamic bone disease both in incident dialysis patients, in prevalently older subjects, or those with a dialytic age of more than two years [13]. This abnormal mineral metabolism currently represents a major therapeutic challenge focusing mainly on the issue of vascular calcifications even in the earlier stages of CKD [14]. Recent evidence has suggested that noncalcium-based phosphate binding agents such as sevelamer hydrochloride, sevelamer carbonate, and lanthanum carbonate may attenuate cardiovascular calcifications compared with calcium-based phosphate binders. Furthermore, as will be discussed subsequently, these agents feature several additional pleiotropic effects including the action exerted on lipid metabolism and systemic inflammatory response.

The aim of the present review is not to illustrate the specific actions produced by calcium-based binders and other drugs, including calcimimetic agents, but rather to focus on the direct and indirect mechanism of action of noncalcium phosphate binders. In doing so, emphasis will be placed almost exclusively to recent studies undertaken and no reference was made to morbidity and mortality rates, which are beyond the scope of this paper.

\section{Phosphate and Actions Produced on Vascular Calcifications}

The recently published KDIGO guidelines, as well as the previous KDOQI version, underline the importance of maintaining phosphate levels within the normal range from the earliest stages of CKD. This recommendation is based on the action exerted by elevated blood phosphate levels in inducing parathyroid cell proliferation and SHPT [15], resulting in an increase in the number of hospitalizations and in mortality rates, bone fractures, and vascular calcifications. Hyperphosphatemia has been associated with a poor outcome and mortality in CKD stage 5D and high normal serum phosphorus levels; CKD patients have the same problem until stage 3. Patients with CKD stages 4-5D frequently feature high serum phosphorus levels linked to the development of aspects of CKD-MBD, including SHPT, reduced serum calcitriol levels, abnormal bone remodelling, and soft-tissue calcifications. Laboratory-based experimental data suggest that hyperphosphatemia may directly cause or exacerbate other aspects of CKD-MBD, specifically arterial calcification. Beta-glycerophosphate, a phosphate donor, and uremic sera induce calcification and osteopontin expression in bovine vascular smooth muscle (BVSM) cells [16]. In vitro experiments have demonstrated how phosphate acts directly on cultured vascular cells to produce an osteochondrogenic phenotypic transformation associated to loss of contractility, mineralization of the extracellular matrix by means of a sodium-dependent phosphate cotransport, expression of specific bone markers, and calcification of matrix protein [17]; a state of microinflammation results in a worsening of this mechanism [18]. It should also be borne to mind that vascular calcification is characterized by the deposition of $\mathrm{Ca}^{2+}$ and inorganic phosphate $\left(\mathrm{P}_{\mathrm{i}}\right)$ in the form of hydroxyapatite (HA) crystals within the medial or neointimal layers of blood vessels. This can involve induction of osteochondrogenic programs of gene expression in vascular smooth muscle cells (VSMCs) [19]. The uremic sera-induced osteopontin expression in BVSMC is partially mediated through alkaline phosphatase activity and an $\mathrm{Na} / \mathrm{Pi}$ cotransporter-dependent mechanism. The association of coronary arteries, the descending aorta, and the mitral annulus irrespective of the presence of other risk factors has previously been demonstrated for the intermediate stages of CKD by findings obtained in the MESA (Multi-Ethnic Study of Atherosclerosis) [20]. Recently, Sage et al. showed that vascular calcifications produced as a result of uremic hyperphosphatemia are associated with vascular cell expression of osteogenic genes, including bone morphogenetic protein- (BMP-) 2 and osteopontin (OPN). High inorganic phosphate levels in vitro stimulate the osteogenic conversion of smooth muscle cells; however, the mechanism governing this is not clear. We found that highphosphate medium increased the expression of BMP-2 and OPN in mouse smooth muscle cells in culture. However, this effect was lost in the presence of the mineralization inhibitor, pyrophosphate, suggesting a contribution of calcium phosphate crystals. These findings prompt further research into the potential of pyrophosphate as treatment for vascular calcification in chronic kidney disease patients [21].

\section{Methods Applied in Literature Review}

Two hundred and thirty-seven published papers describing a correlation between hyperphosphatemia in CKD and vascular calcifications were analyzed. Subsequently, papers relating to a total of 9 randomized trials or featuring an effective study design were selected. All studies had focused on the use of non-calcium phosphate binders as a therapeutic option aimed at reducing nonbone, and prevalently vascular, calcifications in a population of 879 patients. The trials taken into consideration are listed in Tables 1 and 2.

\section{Discussion}

In spite of the lack of evidence from randomized controlled trials (RCTs) to demonstrate that a lowering of phosphorus 
TABLE 1: A study analyzing the effect of non-calcium-based phosphate binders.

\begin{tabular}{|c|c|c|c|c|c|c|}
\hline Reference & Study & $\begin{array}{c}\text { Patient } \\
\text { population }\end{array}$ & \multicolumn{2}{|c|}{ Comparison } & Study design & Results \\
\hline Block et al. [15] & RIND & Incident HD & Calcium (55) & Sevelamer (54) & Randomly assigned & $\begin{array}{l}\text { Calcium binders resulted in more } \\
\text { increase in CACS versus sevelamer } \\
\text { at } 18 \text { months }(P=.015) \text {; median } \\
\text { increase in CACS was } 11 \text { times } \\
\text { greater with calcium versus } \\
\text { sevelamer }(P=.01)\end{array}$ \\
\hline Chertow et al. [24] & TTG & Prevalent HD & Calcium (75) & Sevelamer (66) & Randomly assigned & $\begin{array}{l}\text { Calcium binders with greater } \\
\text { percentage of CACS versus } \\
\text { sevelamer at } 26 \text { and } 52 \mathrm{wk} \\
P=.02, P=.04\end{array}$ \\
\hline Qunibi et al. [31] & CARE-2 & Prevalent HD & Calcium (58) & Sevelamer (68) & $\begin{array}{c}\text { Randomly assigned } \\
\text { with atorvastatin to } \\
\text { both binders or } \\
52 \mathrm{wk}\end{array}$ & $\begin{array}{l}\text { Nonsignificative differences in } \\
\text { CACS between calcium and } \\
\text { sevelamer }\end{array}$ \\
\hline Barreto et al. [32] & BRiC & Prevalent HD & Calcium (18) & Sevelamer (11) & Randomly assigned & $\begin{array}{l}\text { Nonsignificative differences in } \\
\text { CACS between calcium and } \\
\text { sevelamer }\end{array}$ \\
\hline Boaz et al. [35] & N/A & Predialysis pts & Calcium (28) & Sevelamer (27) & $\begin{array}{l}\text { Low-phosphorus } \\
\text { diet with both } \\
\text { binders }\end{array}$ & $\begin{array}{l}\text { Increase in TCS with calcium to } \\
\text { baseline }(P=.001) \text {; NS with } \\
\text { sevelamer }\end{array}$ \\
\hline
\end{tabular}

TABLE 2: A study analyzing the effect of non-calcium-based phosphate binders.

\begin{tabular}{|c|c|c|c|c|c|c|}
\hline Reference & Study & $\begin{array}{c}\text { Patient } \\
\text { population }\end{array}$ & \multicolumn{2}{|c|}{ Comparison } & Study design & Results \\
\hline Chue et al. [51] & N/A & Prevalent HD & $\begin{array}{l}\text { Calcium } \\
\text { (13) }\end{array}$ & Lanthanum (17) & Randomly assigned & $\begin{array}{l}\text { Lanthanum reduced progression } \\
\text { of aortic } \mathrm{VC}(P<.001) \text { versus } \\
\text { calcium }\end{array}$ \\
\hline Raggi et al. [25] & N/A & Prevalent HD & $\begin{array}{l}\text { Calcium } \\
\text { (130) }\end{array}$ & Sevelamer (132) & $\begin{array}{c}\text { Observation at } \\
52 \mathrm{wk}\end{array}$ & $\begin{array}{l}\text { Sevelamer arrested the progression } \\
\text { of valvular and vascular } \\
\text { calcification }(P=.02) \text { versus } \\
\text { calcium }\end{array}$ \\
\hline Galassi et al. [28] & N/A & Incident HD & $\begin{array}{c}\text { Calcium } \\
(20 \mathrm{ND}, 25 \mathrm{D})\end{array}$ & $\begin{array}{c}\text { Sevelamer } \\
(13 \mathrm{ND}, 27 \mathrm{D})\end{array}$ & Randomly assigned & $\begin{array}{l}\text { Hemodialysis diabetic at } 18 \\
\text { months, a greater progression of } \\
\text { CACS }(P=.005) \text { than with } \\
\text { sevelamer with iPTH ranges } \\
\text { suggestive of low bone turnover }\end{array}$ \\
\hline Takei et al. [45] & N/A & Prevalent HD & $\begin{array}{l}\text { Calcium } \\
\text { (21) }\end{array}$ & Sevelamer (21) & Randomly assigned & $\begin{array}{l}\text { Sevelamer suppresses progressive } \\
\text { aortic calcification more than with } \\
\text { calcium }\end{array}$ \\
\hline Raggi et al. [34] & SUMMER & Prevalent HD & $\begin{array}{l}\text { Calcium } \\
\text { (130) }\end{array}$ & Sevelamer (45) & Cross-sectional & $\begin{array}{l}\text { Less carotid and femoral intima } \\
\text { media thickness, minor levels of } \\
\text { LDL-cholesterol, higher iPTH } \\
\text { with sevelamer versus calcium }\end{array}$ \\
\hline
\end{tabular}

levels impacts clinical outcomes, it may be reasonable to lower phosphorus in CKD patients with hyperphosphatemia using phosphate binders. Limiting dietary phosphate intake or increasing the frequency or duration of dialysis sessions is not alone sufficient to lower phosphorus levels. Patient compliance is therefore fundamental not only in limiting dietary intake of phosphorus, but also in adhering to the prescribed protocol of phosphate binder administration, frequently linked to the number of pills the patient is required to take and the degree of gastric tolerance of the product prescribed.

There is insufficient evidence that any specific phosphate binder significantly impacts patient-level outcomes. Thus, the choice of phosphate binder should be selected on an individual basis. Moreover, the lowering of phosphate levels is becoming increasingly acknowledged as mandatory in reducing the onset of nonbone, and in particular cardiac and/or vascular, calcifications that produce a marked 
effect on mortality rates in patients at various stages of CKD.

Large epidemiological studies have consistently shown the importance of hyperphosphatemia as a predictor of mortality in CKD stage 5 patients receiving dialysis. Experimental data suggest a direct causal relationship between phosphorus and several components of CKD-MBD, specifically SHTP bone abnormalities and calcitriol deficiency. Mention is also made of the negative interferences produced by a vitamin $\mathrm{K}$ deficit in patients undergoing dialysis, that may facilitate the onset of vascular calcifications, particularly in patients taking dicumarol [22].

In the present paper we will focus mainly on vascular calcifications and how to personalize treatment with phosphate binders in an attempt to reduce the presence of calcifications on blood vessel walls. The establishing of optimum calcium levels which, if associated to hyperphosphatemia, may give rise to vast and extensive areas of nonbone calcifications is of paramount importance. Non-calcium phosphate binders available currently are aluminium hydroxide, ferric citrate, magnesium carbonate, sevelamer $\mathrm{HCl}$ and sevelamer carbonate, and lanthanum carbonate. This paper will focus mainly on the latter two due to the lack of controlled, prospective, or retrospective trials capable of demonstrating a favourable action of aluminium hydroxide (the use of which is not advised in the long term), magnesium carbonate and ferric citrate on vascular calcifications. Furthermore, studies performed on the dialysis population with niacin and nicotinamide have shown significant phosphate reduction with lowering of the calcium-phosphorus product. However, to date, no published reports or trials have been made available that demonstrate activity of this phosphate binder on vascular calcifications.

\section{Sevelamer from CKD Stage 5D: Effects and Comparison with Calcium-Based Phosphate Binders}

As reported in multiple publications, numerous trials undertaken to compare sevelamer $\mathrm{HCl}$ with calcium-based binders have assessed the impact of phosphate-binder therapy on arterial calcification, assessed using computerized tomography imaging techniques. In two subsequent papers, the first reporting on 200 patients randomized to administration of calcium acetate or sevelamer over a one-year period to correct hyperphosphatemia, and the second paper studying 108 hemodialysis patients likewise randomized to calcium acetate or sevelamer and followed for one year, Chertow demonstrated how calcium treatment led to progressive coronary artery and aortic calcification as determined by sequential EBCT, while the use of the non-calciumcontaining binder sevelamer was devoid of a similar effect $[23,24]$. A further paper examined the effect of these two oral phosphate-binders on valvular calcification in 132 hemodialysis patients, concluding that after a one-year observation period, sevelamer had checked the progression of vascular and valvular calcifications in almost $50 \%$ of patients [25].
Another report suggested that patients randomized to receive calcium salts, compared with those randomized to sevelamer $\mathrm{HCl}$, experienced greater trabecular (but not cortical) bone loss on the basis of changes in thoracic bone mineral density (BMD) on EBCT scans in a subset of 132 patients in whom the necessary imaging was available [26].

More recent paper have expressed some doubt as to the effective harmfulness of the use of calcium-based phosphate binders in the treatment of hyperphosphatemia and vascular calcifications, which has inevitably led to a rise in the use of sevelamer [27]. An interesting study [28] examining the clinical outcomes in a smaller group of 109 incident HD patients (patients new to dialysis, 64 diabetics and 45 non diabetics) randomized to either sevelamer $\mathrm{HCl}$ or calciumbased binder followed up patients for a period of 18 months. Patients received baseline, six, 12, and 18 months EBCT scans. The study found that diabetic patients who used calcium-based phosphate binders experienced more rapid and more severe progression of coronary artery calcification than those using sevelamer.

Diabetic patients treated with calcium-based binders showed a significantly greater coronary artery calcium score (CACS) progression than sevelamer-treated patients (median increase 177 versus 27; $P=.05$ ). During followup, diabetic patients receiving calcium-based binders were significantly more likely to develop serum iPTH values $<16 \mathrm{pmol} / \mathrm{L}$ than diabetic patients treated with sevelamer (33\% versus $6 \%, P=.005)$ and had a lower mean iPTH level $(24 \pm 16$ versus $31 \pm 14 \mathrm{pmol} / \mathrm{L} ; P=.038)$. No data on cardiovascular events other than death, fractures, or parathyroidectomy rates were available from either of these studies, making it impossible to draw conclusions on the impact of using sevelamer $\mathrm{HCl}$ instead of a calciumbased phosphate binder on such outcomes. In addition, no studies have examined the effects of lanthanum carbonate or indeed any other phosphate binder (including calcium- and aluminium-based compounds) on patient-level outcomes.

The largest of these studies, the Dialysis Clinical Outcomes Revisited (DCOR) study, [29] randomized 2103 prevalent $\mathrm{CKD}$ stage $5 \mathrm{D}$ patients to either sevelamer $\mathrm{HCl}$ or a calcium-based phosphate binder $(70 \%$ calcium acetate or $30 \%$ calcium carbonate). The trial was designed to evaluate all-cause mortality; more patients discontinued because of adverse events (AEs) in the sevelamer- $\mathrm{HCl}$ arm ( 8 versus $5 \%$ ), but types of events and event rates were not comprehensively reported. The study was extended because the mortality rate in the control group was lower than expected. Only 1068 patients completed the study, and there were no differences in all-cause or cause-specific mortality rates when comparing sevelamer $\mathrm{HCl}$ (mortality rate 15.0 per 100 patient-years) with calcium-treated patients (16.1 per 100 patient-years). There were also no differences in cardiovascular mortality and hospitalization on the basis of data from case report forms. A possible bias resulted from the limited (90 days) followup of discontinued patients, as well as a lack of documentation of baseline biochemical parameters and AEs.

A secondary analysis of the DCOR study [30] on overall mortality (primary outcome), cause-specific mortality, 
morbidity, or first or cause-specific hospitalization (secondary outcomes) reported how the study had not been well-balanced due to the presence of major atherosclerotic cardiovascular calcifications in patients treated with calciumbased phosphate binders. This study showed a beneficial effect of sevelamer $\mathrm{HCl}$ on the secondary outcomes of multiple all-cause hospitalizations and on the number of days of hospitalization. Furthermore, hospitalizations from CVD as ascertained from the administrative data did not differ, lending no support to the study's hypothesis that sevelamer $\mathrm{HCl}$ reduces cardiovascular disease (CVD) morbidity. The analysis by Peter et al. using claims data described a higher rate of hospitalization in a smaller group of patients with a shorter duration of follow-up than that reported by Suki et al., as a result of the fact that the denominator for hospitalization rates did not include days spent in hospital. Thus, although both analyses showed a trend toward lower hospitalization rates, the fact that the difference between patients allocated to different binders was of statistical significance in the analysis by Peter et al. was not considered robust.

In the CARE 2 study [31], chronic HD patients from the United States were randomized to receive either calcium acetate or sevelamer $\mathrm{HCl}$. Two hundred and eighty-seven patients in both groups received atorvastatin to achieve a low-density lipoprotein cholesterol goal of $70 \mathrm{mg} / \mathrm{dL}$ $(1.81 \mathrm{mmol} / \mathrm{L})$. The study was designed to assess noninferiority, evaluating CAC using EBCT at 6 and 12 months after randomization. Before 1 year, $30 \%$ of the patients in the sevelamer arm and $43 \%$ in the calcium acetate arm dropped out. Despite achieving comparable levels of serum cholesterol, no difference in the progression of arterial calcification was noted when comparing the two treatment arms (calcium acetate and sevelamer $\mathrm{HCl}$ groups, resp., $P=.90)$. The weakness of this study is constituted by the high presence of pre-existing calcifications in the patients investigated.

In a prospective study undertaken by Barreto et al. [32], 101 hemodialysis patients were randomized for each phosphate binder and submitted to multislice coronary and bone biopsies at entry and 12 months to compare the effects of calcium acetate and sevelamer on coronary calcification (CAC) and bone histology; there was no difference in CAC progression or changes in bone remodelling between the calcium and the sevelamer groups; however, this study was hampered by small sample size, use of low dialysate calcium concentrations $(1.25 \mathrm{mmol} / \mathrm{L})$, and no limits in the use of aluminium hydroxide, which likely affected the progression of vascular calcifications. Similar conclusions on CACS and mortality rates were reached by a recent metaanalysis of 2873 patients [33]. A recent paper performed by Raggi et al. [34] of a ten-year period of observation provided encouraging results for the use of sevelamer due to the improvements produced in vascular calcifications, bone physiology, and a potential positive trend in mortality rates for hemodialysis patients. Recently, Boaz et al. [35] studied a group of 45 hemodialysis patients treated with sevelamer and 130 untreated subjects; patients receiving sevelamer had significantly lower carotid intima media thickness and lower low-density lipoprotein cholesterol levels.

Scarce literature reports are available that relate to the predialysis stage of CKD. Relatively few papers have been published, including an Italian study involving 90 bindernaive Italian patients with CKD stages 3-5 who were not receiving dialysis. Russo et al. [36] randomized patients (30 per group) into either a low-phosphate diet alone group, a low-phosphate diet in combination with fixed doses of calcium carbonate $(2 \mathrm{~g} / \mathrm{d})$ group, or a low-phosphate diet in combination with sevelamer $\mathrm{HCl}(1600 \mathrm{mg} / \mathrm{d})$ group and followed up these individuals for 2 years. Coronary artery calcification (CAC) was the primary end point of the study; in the 84 patients who completed the study, the final CAC scores were greater than the initial scores in those receiving diet alone $(P<.001)$ or diet in combination with calcium carbonate $(P<.001)$, whereas there was no progression of calcification in the diet-plus-sevelamer-HCl-treated group.

\section{Mechanisms of Action}

The mechanism through which non-calcium phosphate binders exert their action and slow down progression of vascular calcifications is still unclear and remains to be clarified. However, the RIND study, evaluating progression of coronary artery calcification in incident hemodialysis patients, demonstrated that vascular calcification processes manifest in predialysis stages in the majority of patients, which may well be linked to deranged calcium and phosphate homeostasis. Novel insights into the pathophysiology of calcium and phosphate handling, especially the discovery of the phosphatonin FGF23, suggest that a more complex assessment of phosphate balance is warranted. This assessment should include measurements of fractional phosphate excretion and phosphatonin levels to objectively judge and effectively correct phosphate overload [37].

New markers, such as FGF-23, have been identified as inducers of vascular calcification and cardiovascular disease in CKD. Therefore, the use of calcium-free phosphate binders may reduce the risk of cardiovascular disease by reducing both serum phosphate and FGF-23 levels [38]. Recent findings have provided evidence of a direct action of non-calcium phosphate binders on the progression of vascular calcifications. Data published to date on the use of non-calcium phosphate binders such as sevelamer and lanthanum carbonate recommend the administration of these drugs from the initial stages of CKD in association with a dietary intake of phosphorous in order to prevent an increase in FGF-23 and the action produced by the later on vascular calcifications $[39,40]$.

In view of the large number of reports published on the potential actions exerted by sevelamer in halting the progression of vascular calcifications, this binder should be dealt with separately [41-45]; it has accordingly been demonstrated that sevelamer is capable of lowering mean levels of HDL cholesterol, apolipoprotein B, and homocysteine. Furthermore, a decrease in the expression of the 
nitrotyrosine marker of inflammatory stress by atherosclerotic plaques in the aorta and stabilization of the plaques, a decrease in CRP, and a reduced gastrointestinal absorption of several uremic toxins have also been reported [46]. In addition to the effects produced on FGF23, it is of considerable interest that sevelamer has been found to reduce serum Fetuin A levels, known to exert a cardiovascular protective action [47].

A carbonated form of sevelamer featuring a similar efficacy in control of hyperphosphatemia and which does not worsen metabolic acidosis, as occurs with the $\mathrm{HCl}$ preparation, has recently been introduced onto the market $[48,49]$. At the current time, no conclusive data are available from controlled trials on the action of sevelamer carbonate. It does however seem feasible that this product may possess a similar efficacy in the treatment of vascular calcifications in patients at all stages of CKD [50]. Trials have recently been set up to demonstrate the potential positive effects of lanthanum carbonate on myocardial function and blood vessel elasticity and calcifications [51].

\section{Lanthanum Carbonate}

A positive action of lanthanum carbonate on vascular calcifications had already been hypothesized [52]; although relatively few papers have been published to date to this regard, indications are provided of a positive effect of lanthanum carbonate on the progression of vascular calcifications. One of the most recent papers [53] reported on the findings of a pilot randomized controlled trial undertaken to determine the effect of lanthanum carbonate (LC) on vascular calcification (VC). Forty-five HD patients were randomized to either LC or calcium carbonate. The primary outcome of changes to aortic VC after 18 months included superficial femoral artery (SFA) VC, bone mineral density (BMD) of lumbar spine, and serum markers of mineral metabolism. At baseline, 6-month computed tomography was performed to measure VC and BMD. A random and at 18-month effect linear regression model was performed to assess differences. Results. Thirty patients completed the study; indeed, the small sample size and loss to followup were one of the major limitations to this study. Lanthanum carbonate was associated with the reduced progression of aortic calcification compared with calcium carbonate in HD patients over 18 months.

\section{Magnesium Carbonate}

For the sake of thoroughness, this paper on the action of non-calcium phosphate binders will conclude with a brief analysis of magnesium carbonate and another non-calcium binder was used only rarely. Indeed, an inverse correlation between magnesium levels and the extent of vascular calcifications was demonstrated many years ago (studies from 1974 to 2004) in one study undertaken to evaluate peritoneal dialysis and three conducted on hemodialysis patients; the latter trials have been amply analyzed in a review by Wei et al. in 2006 [54]. Observational data suggest that elevated magnesium levels in dialysis patients may prevent vascular calcification, and in vitro magnesium can prevent hydroxyapatite crystal growth. However, the effects of magnesium on vascular calcification and bone mineral density have not been studied prospectively. In a preliminary study, Spiegel [55] hypothesized that magnesium carbonate was capable of interrupting the progression of vascular calcifications; in a recent open-label pilot study, the same Author studied seven chronic hemodialysis patients to evaluate the effects of a magnesium-based phosphate binder on coronary artery calcification (CAC) scores and vertebral bone mineral density [56]. Magnesium carbonate/calcium carbonate administered as the principal phosphate binder for a period of 18 months and changes in CAC and V-BMD were measured at baseline, 6, 12, and 18 months. Electron beam computed tomography results demonstrated a small and not statistically significant increase in absolute CAC scores, no significant change in median percent change, and a small insignificant change in $\mathrm{V}$-BMD.

To conclude, a large number of reports present in literature recommend that serum phosphate levels be closely monitored, whilst at the same time not overlooking important aspects such as eliciting a decrease in the onset and progression of vascular calcifications that produce a marked effect on the morbidity and mortality rates of CKD patients at all stages. The tendency is to reduce calcium intake both through dialysis and by administration of non-calcium phosphate binders that have proved effective in controlling hyperphosphatemia but which also feature pharmacological characteristics capable of producing a direct decrease in calcifications to the vascular endothelium. In spite of the large body of evidence provided in literature, further randomized, prospective studies should be undertaken with the specific aim of evaluating the incident population to provide further confirmation and optimization of the beneficial effects reported to date.

\section{References}

[1] G. M. London, A. P. Guerin, S. J. Marchais et al., "Cardiac and arterial interactions in end-stage renal disease," Kidney International, vol. 50, no. 2, pp. 600-608, 1996.

[2] J. Blacher, B. Pannier, A. P. Guerin, S. J. Marchais, M. E. Safar, and G. M. London, "Carotid arterial stiffness as a predictor of cardiovascular and all- cause mortality in end-stage renal disease," Hypertension, vol. 32, no. 3, pp. 570-574, 1998.

[3] J. Blacher, A. P. Guerin, B. Pannier, S. J. Marchais, M. E. Safar, and G. M. London, "Impact of aortic stiffness on survival in end-stage renal disease," Circulation, vol. 99, no. 18, pp. 24342439, 1999.

[4] A. Y. M. Wang, M. Wang, J. Woo et al., "Cardiac valve calcification as an important predictor for all-cause mortality and cardiovascular mortality in long-term peritoneal dialysis patients: a prospective study," Journal of the American Society of Nephrology, vol. 14, no. 1, pp. 159-168, 2003.

[5] P. Raggi, "Role of electron-beam computed tomography and nuclear stress testing in cardiovascular risk assessment," American Journal of Cardiology, vol. 96, no. 8, pp. 20J-27J, 2005. 
[6] M. Cozzolino, D. Brancaccio, M. Gallieni, and E. Slatopolsky, "Pathogenesis of vascular calcification in chronic kidney disease," Kidney International, vol. 68, no. 2, pp. 429-436, 2005.

[7] "KDIGO Clinical Practice Guideline for the Diagnosis, Evaluation, Prevention, and Treatment of Chronic Kidney Disease-Mineral and Bone Disorder (CKD-MBD). Chapter 4.1: treatment of CKD-MBD targeted at lowering high serum phosphorus and maintaining serum calcium," Kidney International, vol. 76, supplement 113, pp. S50-S99, 2009.

[8] C. Zehnder, J. P. Gutzwiller, and K. Renggli, "Hemodiafiltration-a new treatment option for hyperphosphatemia in hemodialysis patients," Clinical Nephrology, vol. 52, no. 3, pp. $152-159,1999$.

[9] W. Lornoy, J. De Meester, I. Becaus, J. M. Billiouw, P. A. Van Malderen, and M. Van Pottelberge, "Impact of convective flow on phosphorus removal in maintenance hemodialysis patients," Journal of Renal Nutrition, vol. 16, no. 1, pp. 47-53, 2006.

[10] L. A. Pedrini, V. De Cristofaro, M. Comelli et al., "Longterm effects of high-efficiency on-line haemodiafiltration on uraemic toxicity. A multicentre prospective randomized study," Nephrology Dialysis Transplantation. In press.

[11] F. Gotch, N. W. Levin, and P. Kotanko, "Calcium balance in dialysis is best managed by adjusting dialysate calcium guided by kinetic modeling of the interrelationship between calcium intake, dose of vitamin D analogues and the dialysate calcium concentration," Blood Purification, vol. 29, no. 2, pp. 163-176, 2010.

[12] M. A. Mason and B. M. Shepler, "Evaluation of morbidity and mortality data related to cardiovascular calcification from calcium-containing phosphate binder use in patients undergoing hemodialysis," Pharmacotherapy, vol. 30, no. 7, pp. 741-748, 2010.

[13] J. M. Frazão and T. Adragão, "Treatment of hyperphosphatemia with sevelamer hydrochloride in dialysis patients: effects on vascular calcification, bone and a close look into the survival data," Kidney International. Supplement, no. 111, pp. S38-43, 2008.

[14] S. M. Moe, T. Drüeke, N. Lameire, and G. Eknoyan, "Chronic kidney disease-mineral-bone disorder: a new paradigm," Advances in Chronic Kidney Disease, vol. 14, no. 1, pp. 3-12, 2007.

[15] G. A. Block, T. E. Hulbert-Shearon, N. W. Levin, and F. K. Port, "Association of serum phosphorus and calcium x phosphate product with mortality risk in chronic hemodialysis patients: a national study," American Journal of Kidney Diseases, vol. 31, no. 4, pp. 607-617, 1998.

[16] N. X. Chen, K. D. O’Neill, D. Duan, and S. M. Moe, "Phosphorus and uremic serum up-regulate osteopontin expression in vascular smooth muscle cells," Kidney International, vol. 62, no. 5, pp. 1724-1731, 2002.

[17] S. Jono, A. Shioi, Y. Ikari, and Y. Nishizawa, "Vascular calcification in chronic kidney disease," Journal of Bone and Mineral Metabolism, vol. 24, no. 2, pp. 176-181, 2006.

[18] C. M. Giachelli, "The emerging role of phosphate in vascular calcification," Kidney International, vol. 75, no. 9, pp. 890-897, 2009.

[19] V. P. Iyemere, D. Proudfoot, P. L. Weissberg, and C. M. Shanahan, "Vascular smooth muscle cell phenotypic plasticity and the regulation of vascular calcification," Journal of Internal Medicine, vol. 260, no. 3, pp. 192-210, 2006.

[20] J. H. Ix, I. H. De Boer, C. A. Peralta et al., "Serum phosphorus concentrations and arterial stiffness among individuals with normal kidney function to moderate kidney disease in MESA," Clinical Journal of the American Society of Nephrology, vol. 4, no. 3, pp. 609-615, 2009.

[21] A. P. Sage, J. Lu, Y. Tintut, and L. L. Demer, "Hyperphosphatemia-induced nanocrystals upregulate the expression of bone morphogenetic protein-2 and osteopontin genes in mouse smooth muscle cells in vitro," Kidney International, vol. 79, no. 4, pp. 414-422, 2011.

[22] T. Krueger, R. Westenfeld, M. Ketteler, L. J. Schurgers, and J. Floege, "Vitamin K deficiency in CKD patients: a modifiable risk factor for vascular calcification?" Kidney International, vol. 76, no. 1, pp. 18-22, 2009.

[23] G. M. Chertow, S. K. Burke, and P. Raggi, "Sevelamer attenuates the progression of coronary and aortic calcification in hemodialysis patients," Kidney International, vol. 62, no. 1, pp. 245-252, 2002.

[24] G. M. Chertow, P. Raggi, J. T. McCarthy et al., "The effects of sevelamer and calcium acetate on proxies of atherosclerotic and arteriosclerotic vascular disease in hemodialysis patients," American Journal of Nephrology, vol. 23, no. 5, pp. 307-314, 2003.

[25] P. Raggi, J. Bommer, and G. M. Chertow, "Valvular calcification in hemodialysis patients randomized to calcium-based phosphorus binders or sevelamer," The Journal of Heart Valve Disease, vol. 13, no. 1, pp. 134-141, 2004.

[26] P. Raggi, G. James, S. K. Burke et al., "Decrease in thoracic vertebral bone attenuation with calcium-based phosphate binders in hemodialysis," Journal of Bone and Mineral Research, vol. 20, no. 5, pp. 764-772, 2005.

[27] E. A. Friedman, "Calcium-based phosphate binders are appropriate in chronic renal failure," Clinical Journal of the American Society of Nephrology, vol. 1, no. 4, pp. 704-709, 2006.

[28] A. Galassi, D. M. Spiegel, A. Bellasi, G. A. Block, and P. Raggi, "Accelerated vascular calcification and relative hypoparathyroidism in incident haemodialysis diabetic patients receiving calcium binders," Nephrology Dialysis Transplantation, vol. 21, no. 11, pp. 3215-3222, 2006.

[29] W. N. Suki, R. Zabaneh, J. L. Cangiano et al., "Effects of sevelamer and calcium-based phosphate binders on mortality in hemodialysis patients," Kidney International, vol. 72, no. 9, pp. 1130-1137, 2007.

[30] W. L. S. T. Peter, J. Liu, E. Weinhandl, and Q. Fan, "A Comparison of sevelamer and calcium-based phosphate binders on mortality, hospitalization, and morbidity in hemodialysis: a secondary analysis of the dialysis clinical outcomes revisited (DCOR) randomized trial using claims data," American Journal of Kidney Diseases, vol. 51, no. 3, pp. 445-454, 2008.

[31] W. Qunibi, M. Moustafa, L. R. Muenz et al., "A 1-year randomized trial of calcium acetate versus sevelamer on progression of coronary artery calcification in hemodialysis patients with comparable lipid control: the calcium acetate renagel evaluation-2 (CARE-2) study," American Journal of Kidney Diseases, vol. 51, no. 6, pp. 952-965, 2008.

[32] D. V. Barreto, F. D. C. Barreto, A. B. De Carvalho et al., "Phosphate binder impact on bone remodeling and coronary calcification-results from the BRiC study," NephronClinical Practice, vol. 110, no. 4, pp. c273-c283, 2008.

[33] S. A. Jamal, D. Fitchett, C. E. Lok, D. C. Mendelssohn, and R. T. Tsuyuki, "The effects of calcium-based versus non-calciumbased phosphate binders on mortality among patients with chronic kidney disease: a meta-analysis," Nephrology Dialysis Transplantation, vol. 24, no. 10, pp. 3168-3174, 2009.

[34] P. Raggi, S. Vukicevic, R. M. Moysés, K. Wesseling, and D. M. Spiegel, "Ten-year experience with sevelamer and calcium salts 
as phosphate binders," Clinical Journal of the American Society of Nephrology, vol. 5, no. 1, pp. S31-S40, 2010.

[35] M. Boaz, Z. Katzir, D. Schwartz et al., "Effect of sevelamer hydrochloride exposure on carotid intima media thickness in hemodialysis patients," Nephron-Clinical Practice, vol. 117, no. 2, pp. c83-c88, 2011.

[36] D. Russo, I. Miranda, C. Ruocco et al., "The progression of coronary artery calcification in predialysis patients on calcium carbonate or sevelamer," Kidney International, vol. 72, no. 10, pp. 1255-1261, 2007.

[37] M. Ketteler and P. H. Biggar, "Dietary and pharmacological control of calcium and phosphate metabolism in predialysis stages of chronic kidney disease," Blood Purification, vol. 27, no. 4, pp. 345-349, 2009.

[38] M. Cozzolino, E. Missaglia, A. Ortiz et al., "Vascular calcification in chronic kidney disease," Recenti Progressi in Medicina, vol. 101, no. 11, pp. 442-452, 2010.

[39] R. B. Oliveira, A. L. E. Cancela, F. G. Graciolli et al., "Early control of PTH and FGF23 in normophosphatemic CKD patients: a new target in CKD-MBD therapy?" Clinical Journal of the American Society of Nephrology, vol. 5, no. 2, pp. 286291, 2010.

[40] T. Isakova, O. M. Gutirrez, K. Smith et al., "Pilot study of dietary phosphorus restriction and phosphorus binders to target fibroblast growth factor 23 in patients with chronic kidney disease," Nephrology Dialysis Transplantation, vol. 26, no. 2, pp. 584-591, 2011.

[41] V. Panichi, R. Bigazzi, S. Paoletti et al., "Impact of calcium, phosphate, PTH abnormalities and management on mortality in hemodialysis: results from the RISCAVID study," Journal of Nephrology, vol. 23, no. 5, pp. 556-562, 2010.

[42] M. Ketteler, M. Rix, S. Fan et al., "Efficacy and tolerability of sevelamer carbonate in hyperphosphatemic patients who have chronic kidney disease and are not on dialysis," Clinical Journal of the American Society of Nephrology, vol. 3, no. 4, pp. 11251130, 2008.

[43] J. Delmez, G. Block, J. Robertson et al., "A randomized, double-blind, crossover design study of sevelamer hydrochloride and sevelamer carbonate in patients on hemodialysis," Clinical Nephrology, vol. 68, no. 6, pp. 386-391, 2007.

[44] S. Fan, C. Ross, S. Mitra et al., "A randomized, crossover design study of sevelamer carbonate powder and sevelamer hydrochloride tablets in chronic kidney disease patients on haemodialysis," Nephrology Dialysis Transplantation, vol. 24, no. 12, pp. 3794-3799, 2009.

[45] T. Takei, S. Otsubo, K. Uchida et al., "Effects of sevelamer on the progression of vascular calcification in patients on chronic haemodialysis," Nephron-Clinical Practice, vol. 108, no. 4, pp. c278-c283, 2008.

[46] V. M. Brandenburg, W. Jahnen-Dechent, and M. Ketteler, "Sevelamer and the bone-vascular axis in chronic kidney disease: bone turnover, inflammation, and calcification regulation," Kidney International. Supplement, no. 114, pp. S26-33, 2009.

[47] K. Caglar, M. I. Yilmaz, M. Saglam et al., "Short-term treatment with sevelamer increases serum fetuin-A concentration and improves endothelial dysfunction in chronic kidney disease stage 4 patients," Clinical Journal of the American Society of Nephrology, vol. 3, no. 1, pp. 61-68, 2008.

[48] S. Fishbane, J. Delmez, W. N. Suki et al., "A randomized, parallel, open-label study to compare once-daily sevelamer carbonate powder dosing with thrice-daily sevelamer hydrochloride tablet dosing in CKD patients on hemodialysis," American Journal of Kidney Diseases, vol. 55, no. 2, pp. 307-315, 2010.
[49] M. D. Arenas, P. Rebollo, T. Malek et al., "A comparative study of 2 new phosphate binders (sevelamer and lanthanum carbonate) in routine clinical practice," Journal of Nephrology, vol. 23, no. 6, pp. 683-692, 2010.

[50] R. Wilson, P. Zhang, M. Smyth, and R. Pratt, "Assessment of survival in a 2-year comparative study of lanthanum carbonate versus standard therapy," Current Medical Research and Opinion, vol. 25, no. 12, pp. 3021-3028, 2009.

[51] C. D. Chue, J. N. Townend, R. P. Steeds, and C. J. Ferro, "Evaluating the effects of sevelamer carbonate on cardiovascular structure and function in chronic renal impairment in Birmingham: the CRIB-PHOS randomised controlled trial," Trials, vol. 12, article 30, pp. 1-7, 2011.

[52] M. Cozzolino and D. Brancaccio, "Clinical consequences and novel therapy of hyperphosphatemia: lanthanum carbonate for dialysis patients," Recent Patents on Cardiovascular Drug Discovery, vol. 2, no. 1, pp. 29-34, 2007.

[53] N. D. Toussaint, K. K. Lau, K. R. Polkinghorne, and P. G. Kerr, "Attenuation of aortic calcification with lanthanum carbonate versus calcium-based phosphate binders in haemodialysis: a pilot randomized controlled trial," Nephrology, vol. 16, no. 3, pp. 290-298, 2011.

[54] M. Wei, K. Esbaei, J. Bargman, and D. G. Oreopoulos, "Relationship between serum magnesium, parathyroid hormone, and vascular calcification in patients on dialysis: a literature review," Peritoneal Dialysis International, vol. 26, no. 3, pp. 366-373, 2006.

[55] D. M. Spiegel, "The role of magnesium binders in chronic kidney disease," Seminars in Dialysis, vol. 20, no. 4, pp. 333336, 2007.

[56] D. M. Spiegel and B. Farmer, "Long-term effects of magnesium carbonate on coronary artery calcification and bone mineral density in hemodialysis patients: a pilot study," Hemodialysis International, vol. 13, no. 4, pp. 453-459, 2009. 


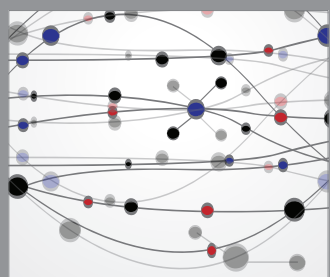

The Scientific World Journal
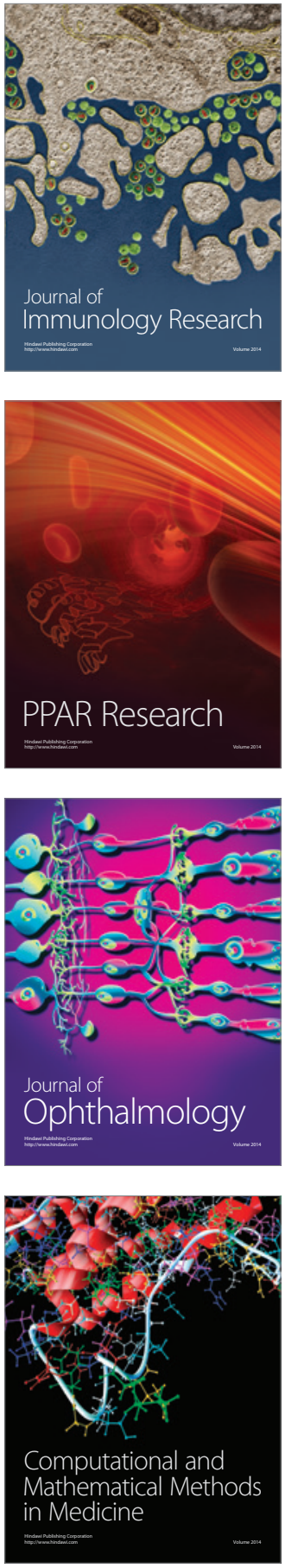

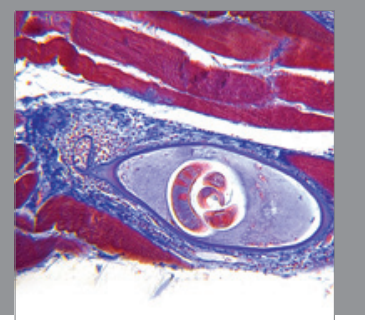

Gastroenterology

Research and Practice
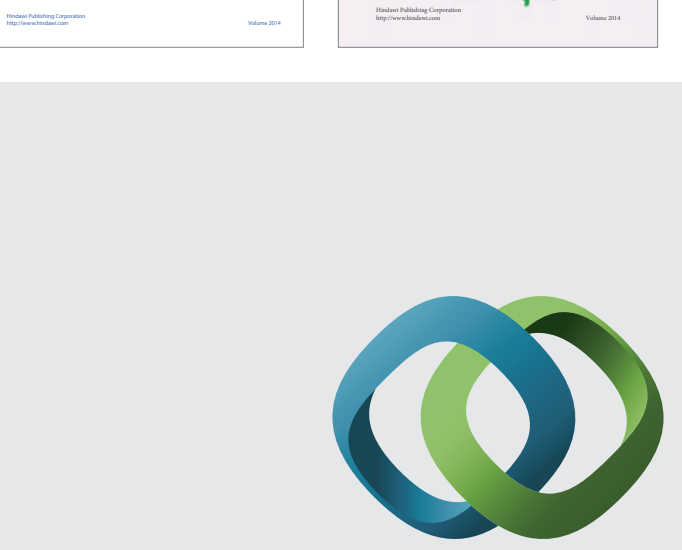

\section{Hindawi}

Submit your manuscripts at

http://www.hindawi.com
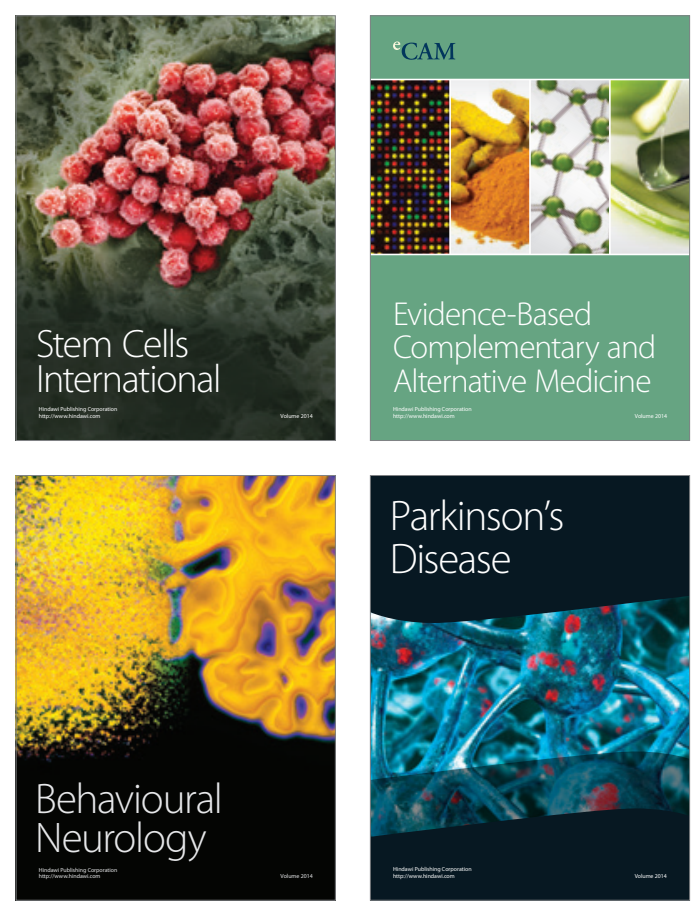

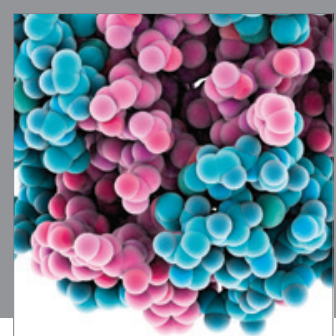

Journal of
Diabetes Research

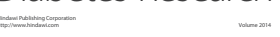

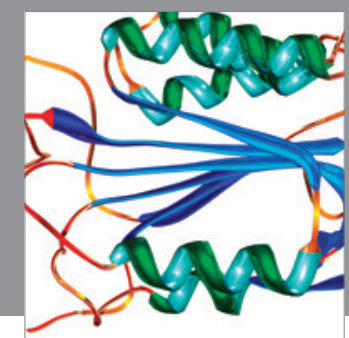

Disease Markers
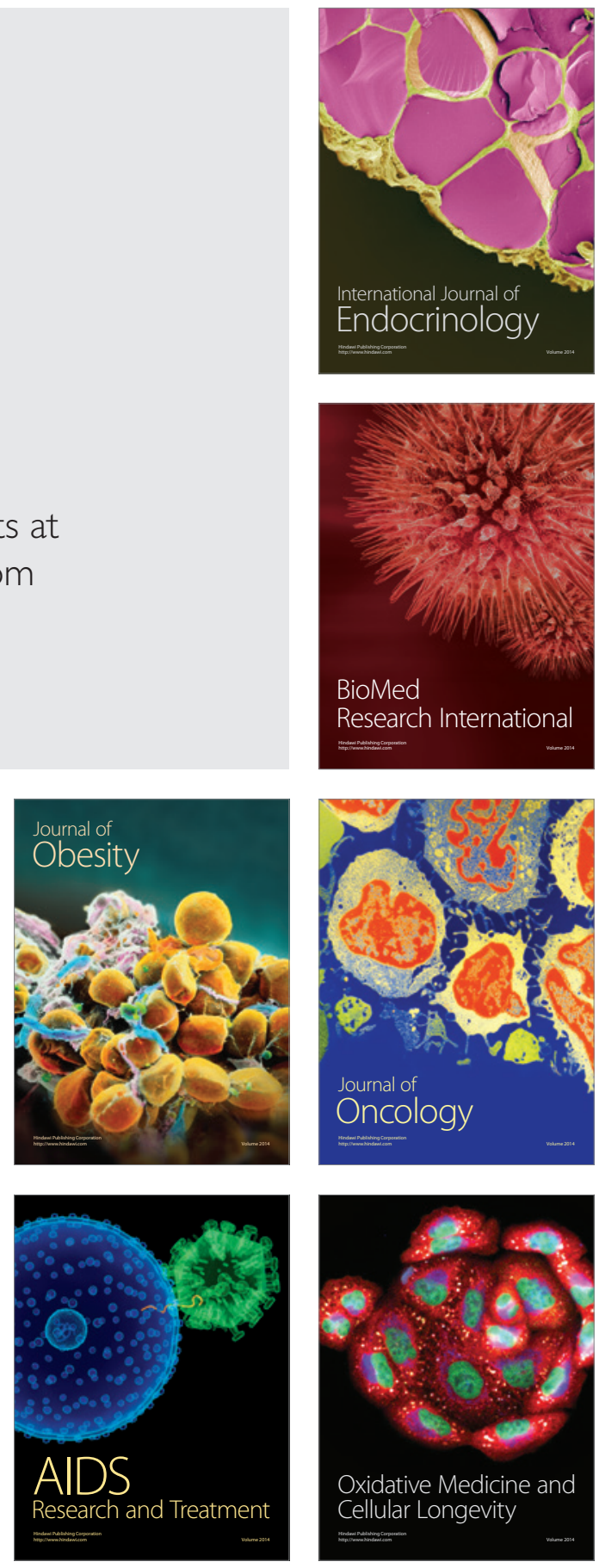